Check for updates

Cite this: Phys. Chem. Chem. Phys., 2020, 22, 4840

DOI: $10.1039 / \mathrm{d} 0 \mathrm{cp} 90040 \mathrm{k}$

rsc.li/pccp

\section{Correction: Conformation of bis-nitroxide polarizing agents by multi-frequency EPR spectroscopy}

\author{
Janne Soetbeer, (D) $\dagger^{a}$ Peter Gast, ${ }^{b}$ Joseph J. Walish, ${ }^{c}$ Yanchuan Zhao, (D) c \\ Christy George, (D) a Chen Yang, ${ }^{a}$ Timothy M. Swager, (D) ${ }^{c}$ Robert G. Griffin (D) a and \\ Guinevere Mathies (D) $\ddagger^{*^{a}}$
}

Correction for 'Conformation of bis-nitroxide polarizing agents by multi-frequency EPR spectroscopy' by Janne Soetbeer et al., Phys. Chem. Chem. Phys., 2018, 20, 25506-25517.

We correct an error in eqn (4) in our published article. The correct expression for the dipolar coupling between two electron spins in the high-field limit is

$$
\boldsymbol{D}^{\mathrm{PAS}, \mathrm{D}}=-\frac{\mu_{0}}{4 \pi \hbar} \frac{g_{1} g_{2} \mu_{\mathrm{B}}^{2}}{r_{12}{ }^{3}}\left(\begin{array}{ccc}
-1 & 0 & 0 \\
0 & -1 & 0 \\
0 & 0 & 2
\end{array}\right)
$$

Thus, a minus-sign was lacking previously. In our calculations the correct expression was used (the minus-sign was present) and, hence, our results and conclusions are unaltered.

We furthermore note that in eqn (1) the exchange interaction, $J$, was erroneously typeset in bold. Our assumption is that the exchange interaction is isotropic and can be represented by a scalar.

The Royal Society of Chemistry apologises for these errors and any consequent inconvenience to authors and readers.

\footnotetext{
${ }^{a}$ Francis Bitter Magnet Laboratory and Department of Chemistry, Massachusetts Institute of Technology, Cambridge, MA 02139, USA

${ }^{b}$ Department of Physics, Huygens-Kamerlingh Onnes Laboratory, Leiden University, PO Box 9504, 2300 RA Leiden, The Netherlands

${ }^{c}$ Department of Chemistry, Massachusetts Institute of Technology, Cambridge, MA 02139, USA

$\dagger$ Current address: Laboratory of Physical Chemistry, Department of Chemistry and Applied Biosciences, ETH Zurich, Vladimir-Prelog-Weg 2, 8093 Zürich, Switzerland.

\# Current address: Department of Chemistry, University of Konstanz, 78464 Konstanz, Germany. E-mail: guinevere.mathies@uni-konstanz.de
} 